ON DISTIRTET COMMUICATIONS NETWORKS

\author{
Paul Baran
}

September 1962

$P-2626$ 



\title{
ON DISTRIBUTED COMMUNICATIONS NETWORKS
}

\author{
Paul Baran* \\ The RAND Corporation, Santa Monica, California
}

\section{INTRODUCTION}

The previous paper** described how redundancy of coding can be used to build efficient digital data links out of transmission links of variable and often less than presently useful quality. An arbitrarily low over-all error rate can be purchased with a modest redundancy of coding and clever terminal equipment. But even links with low error rates can have less than perfect reliability.

We should like to extend the remarks of the previous paper and address ourselves to the problem of building

* Any views expressed in this paper are those of the author. They should not be interpreted as reflecting the views of The RAND Corporation or the official opinion or policy of any of its governmental or private research sponsors. Papers are reproduced by The RAND Corporation as a courtesy to members of its staff.

This paper was prepared for presentation at the First Congress of the Information Systems Sciences, sponsored by The MITRE Corporation and the USAF Electronic Systems Division, November, 1962.

The writer is indebted to John Bower for his suggestions that switching in any store-and-forward system can be described by a model of a postmaster at a blackboard. Programming assistance provided by Sharla Boehm, John Derr, and Joseph Smith is gratefully acknowledged.

**A prior paper was presented by Paul Rosen and Irwin Lebow of MIT Iincoln Laboratorles, discussing redundancy of coding on a single link, "Low Error Efficient Digitial Communications Links," First Congress on the Information Systems Sciences, McGraw-Hill, New York, 1962. 
digital communication networks using links with less than perfect reliability. We shall again trade in the currency of redundancy, but instead of redundancy of coding we shall make use of redundancy of connectivity.

This thing called redundancy is a powerful tool. But the systems planner must choose that form of redundancy so that the form of the "noise" or interference appears to be somewhat statistically independent for each redundant element added. If this goal is completely met, there can be an exponential payoff for a linear increase of added elements. As an example, we shall consider in some detail the synthesis of a system where the form of the disturbance or "noise" is the simultaneous destruction of many geographically separated installations. The system in particular is to be a very high-speed digital data transmission network composed of unreliable links, but which exhibits any arbitrarily desired level of system reliability or survivability.

\section{DEFINITION OF SURVIVABILITY}

This communications network shall be composed of several hundred stations which must intercommunicate with one another. Survivability as herein defined is the percentage of stations surviving a physical attack and remaining in electrical connection with the largest single group of surviving stations. This criterion is a measure of the ability of the surviving stations to operate together as a coherent entity after attack. 
TYPES OF NETWORKS

Although one can draw a wide variety of networks, they all factor into two components: centralized (or star) and distributed (or grid or mesh). (Types (a) and (c) in Fig. 1)

The centralized network is basically vulnerable. Destruction of the central node destroys intercommunication between the end stations. In practice, a mixture of star and mesh components is used to form communications networks. For example, type (b) in Fig. I shows a hierarchial structure to a set of stars connected in the form of a larger star with an additional link forming a loop. Such a network is sometimes called a "decentralized" network, because complete reliance upon a single point is not always required. But, as destruction of a small number of nodes in a decentralized network can destroy communications, we shall turn to consider the properties, problems, and hopes of building communications networks that are as "distributed" as possible. The unstandardized terms centralized, decentralized, and distributed are often and conveniently used as relative adjectives when referring to real-world networks.

\section{DEFINITION OF REDUNDANCY IEVEL}

Figure 2 defines the term "redundancy level," which is used in this paper as a measure of connectivity. A minimum span network, one formed with the smallest number 


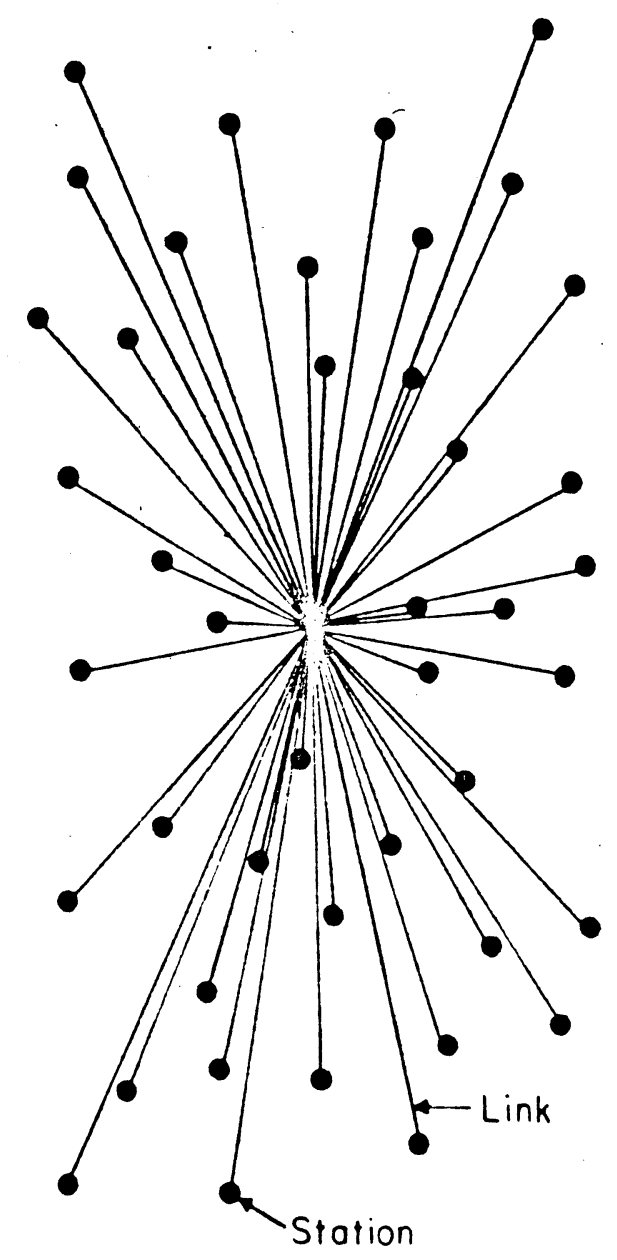

CENTRALIZED

(A)

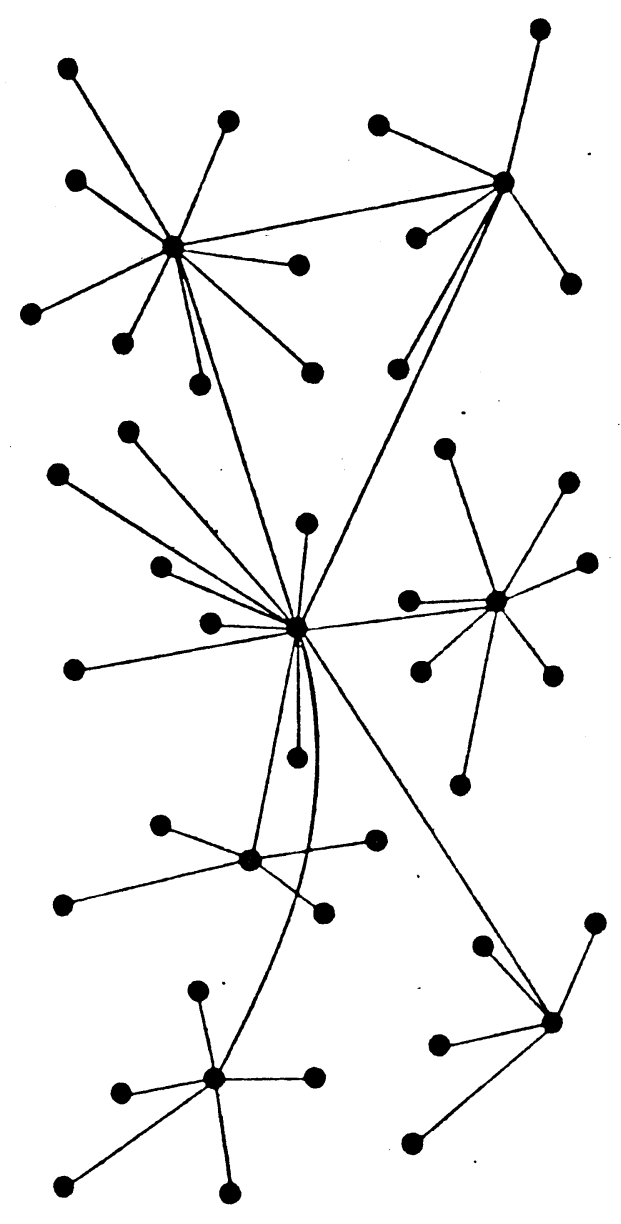

DECENTRALIZED

(B)

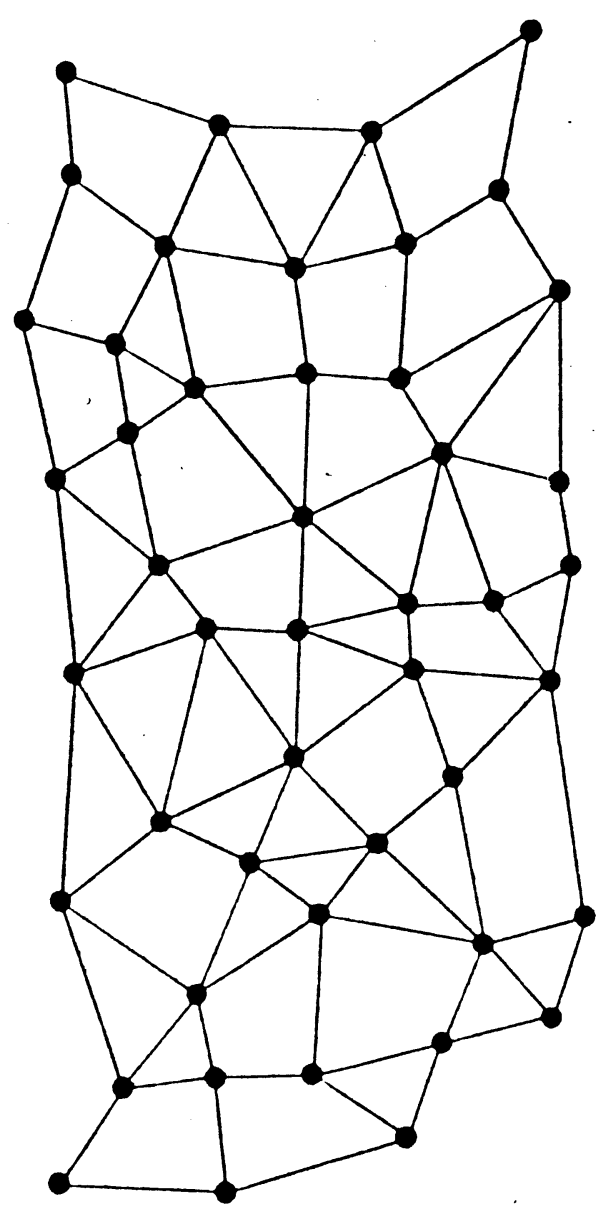

$\frac{1}{1}$

FIG. I - Centralized, Decentralized and Distributed Networks 
of links possible, is chosen as a reference point, and is called "a network of redundancy level one." If two times ? as many links are used in a gridded network than in a minimum span network, the network is said to have a re-

' dundancy level of two. Figure 2 defines connectivity of $1,1 \frac{1}{2}, 2,3,4,6$, and 8 . Redundancy level is equivalent to link-to-node ratio in an infinite size arrays of stations.

\section{ASSUMPTION OF PERFECT SWITCHING}

Each node and link in the array of Fig. 2 has the capacity and the switching flexibility to allow transmission between any ith station and any $j$ th station, provided a path can be drawn from the ith to the jth station.

Starting with a network composed of an array of stations connected as in Fig. 3, an assigned percentage of nodes and links are destroyed. If, after this operation, it is still possible to draw a line to connect the ith station to the $j \underline{t h}$ station, the $i$ th and $j$ th stations are said to be connected.

\section{RATIONALE FOR DESTRUCTION PATTERNS}

Figure 4 indicates network performance as a function of the probability of destruction for each separate node. If the expected "noise" was destruction caused by conventional hardware failure, the failures would be randomly. distributed through the network. But, if the disturbance 

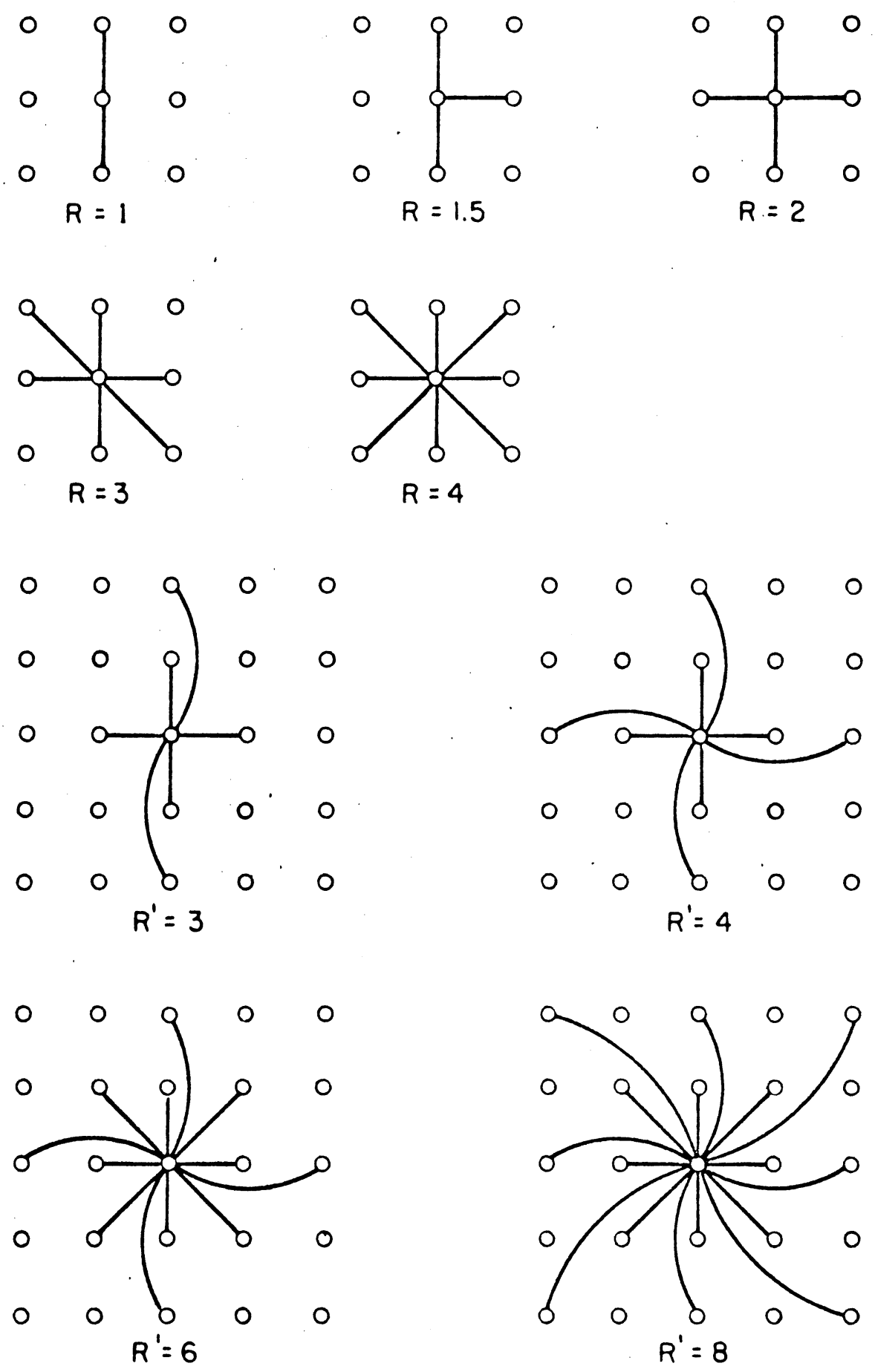

FIG. 2- Definition of Redundancy Level 


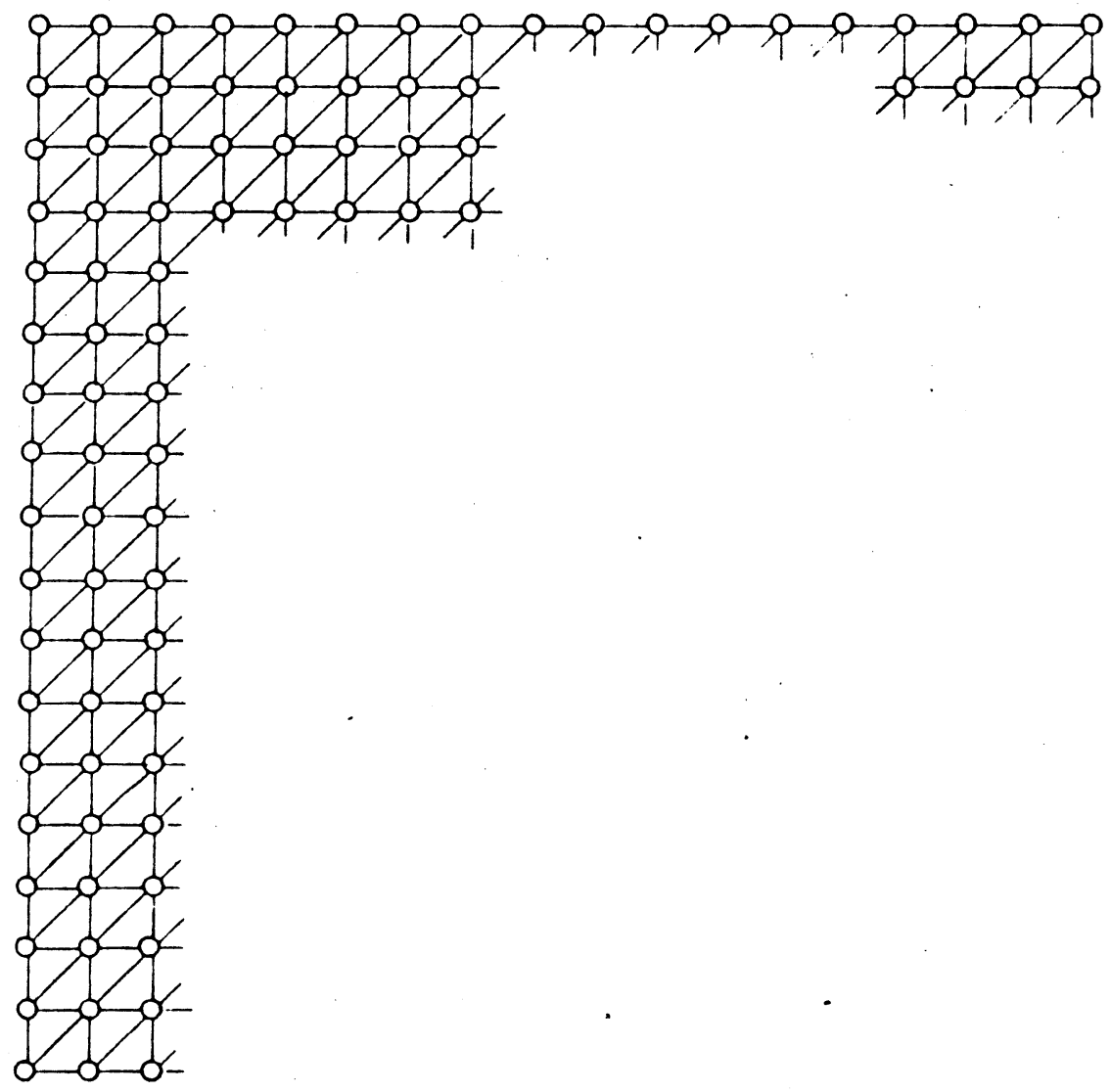

FIG. 3 - An Array of Stations 


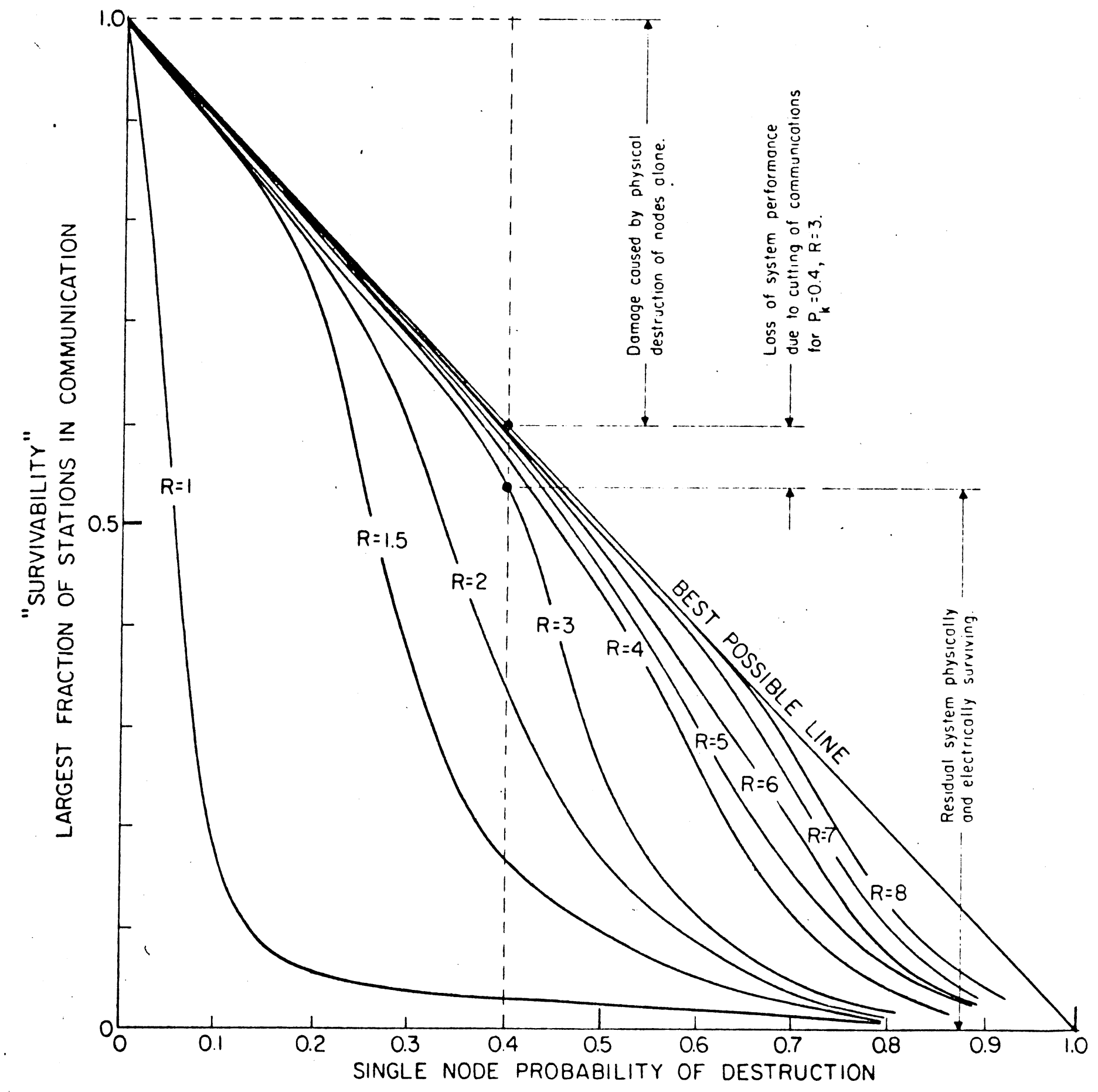

FIG. 4 - Perfect Switching in a Distributed Network - Sensitivity to Node Destruction, $100 \%$ of Links Operative. 
were caused by enemy attack there are two possible "worst cases" to be considered.

To bisect a 32-link width network requires direction of 288 weapons each with a probability of kill, $p_{k}=0.5$, or 160 with a $p_{k}=0.7$, to produce over an 0.9 probability of successfully bisecting the network. If hidden alternative command is allowed, then the largest single group would still have an expected value of almost 50 percent of the initial stations surviving intact. If this raid misjudges complete availability of weapons, or complete knowledge of all links in the cross section, or the effects of the weapons against each and every link, the raid fails. The high risk of such raids against highly parallel structures causes examination of alternative attack policies. Consider the following uniform raid example. Assume that 2,000 weapons are deployed against a 1000-station network. The stations are so spaced that destruction of two stations with a single weapon is unlikely. Divide the 2,000 weapons into two equal 1,000 weapon salvos. Assume any probability of destruction of a single node from a single weapon less than 1.0; for example, 0.5. Each weapon on the first salvo has a 0.5 probability of destroying its target. But, each. weapon of the second salvo has only a 0.25 probability, since one-half the targets have already been destroyed. Thus, the uniform attack is felt to represent a worst-case configuration. 


\section{MONTE CARLO SIMULATION}

Such worst-case attacks have been directed against an 18x18-array network model of 324 nodes with varying probability of $\mathrm{klll}$ and redundancy level, with results shown in Fig. 4. The probability of kill was varied from zero to unity along the abscissa while the ordinate marks survivabilit. The criterion of survivability used is the percentace of stations not physically destroyed and remaining in communications with the larest single group of survivine stations. The curves of Fig. 4 demonstrate survivability as function of attack level for networks of varyin. degrees of redundancy. The line labeled "best possibie line" marks the upper bound of loss due to the physical failure component alone. For example, if a network underwent an attack of 0.5 probability destruction of each of its nodes, then only 50 per cent of its noajes would be expected to survive--regardless of how perfect its communications. We are primarily interested in the aditional system degradation caused by failure of communications. Two key points are to be noticed in the curves of Fie. 4. First, extremely survivable networks can be built using a moderately low redunaancy of connectivity level. Redundancy levels on the order of only three permit withstanding extremely heavy level attacks with negligible additional los to communications. Secondly, the survivability curves have sharp break-points. 
A network of this type will withstand an increasing attack level until a certain point is reached, beyond which the network rapidly deteriorates. Thus, the optimum degree of redundancy can be chosen as a function of the expected level of attack. Further redundancy buys little. The redundancy level required to survive even very heavy attacks is not great--on the order of only three or four times that of the minimum span network.

\section{SIMULATION RESULTS--LINK FAILURE ONLY}

In the previous example we have examined network performance as a function of the destruction of the nodes (which are better targets than links). We shall now reexamine the same network, but using unreliable links. In particular, we want to know how unreliable the links may be without further degrading the performance of the network.

Figure 5 shows the results for the case of perfect nodes; only the links fail. There is little system degradation caused even using extremely unreliable links-on the order of 50 percent down-time--assuming all nodes are working.

COMBINATION LINK AND NODE FAILURES

The worst case is the composite effect of failures of both the links and the nodes. Figure 6 shows the effect of link failure upon a network having 40 percent of its nodes destroyed. It appears that what would today be regarded as an unreliable link can be used in a distributed 


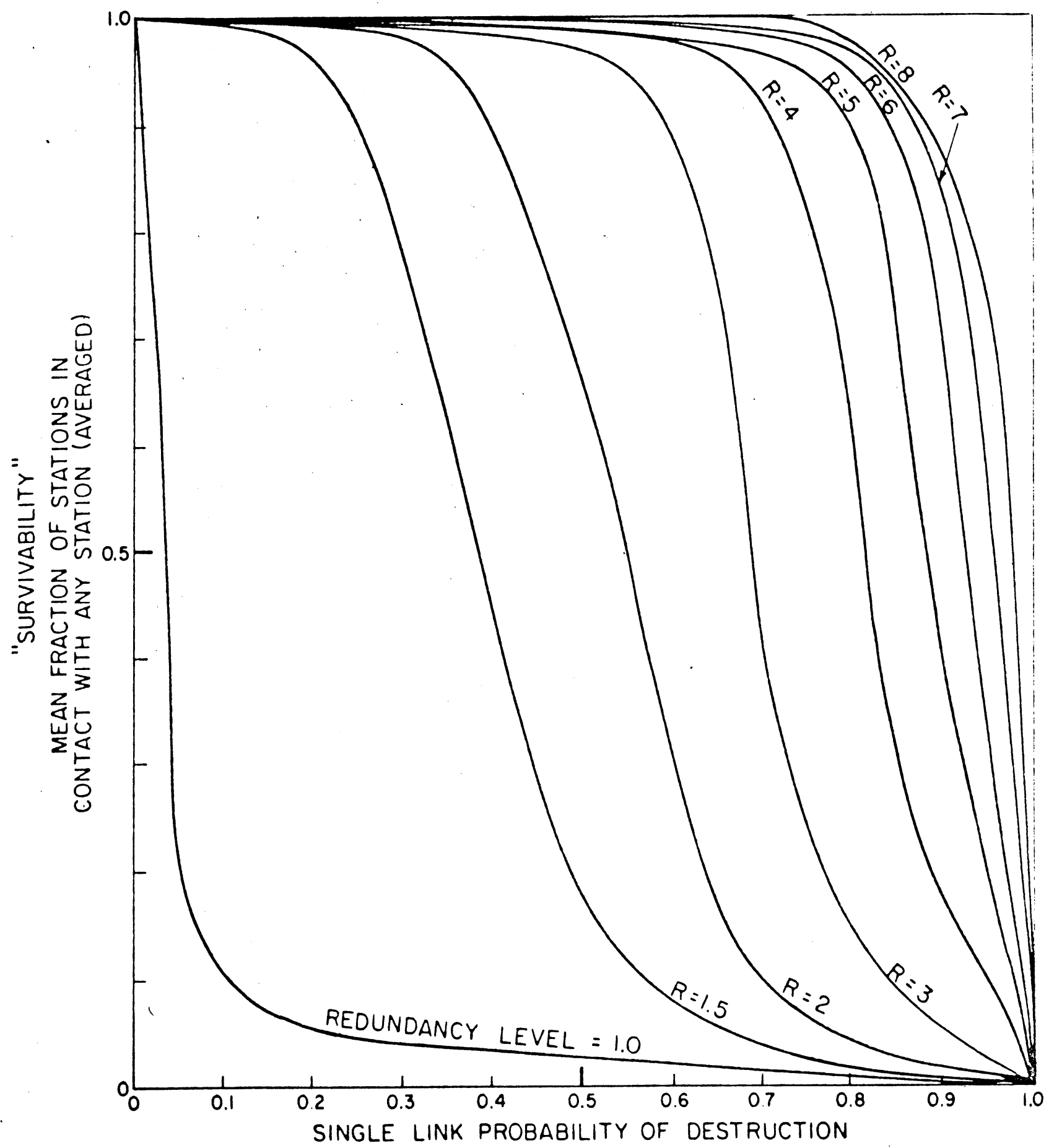

FIG. 5 - Perfect Switching in a Distributed Network - Sensitivity to Link Destruction, 100\% of Nodes Operative. 


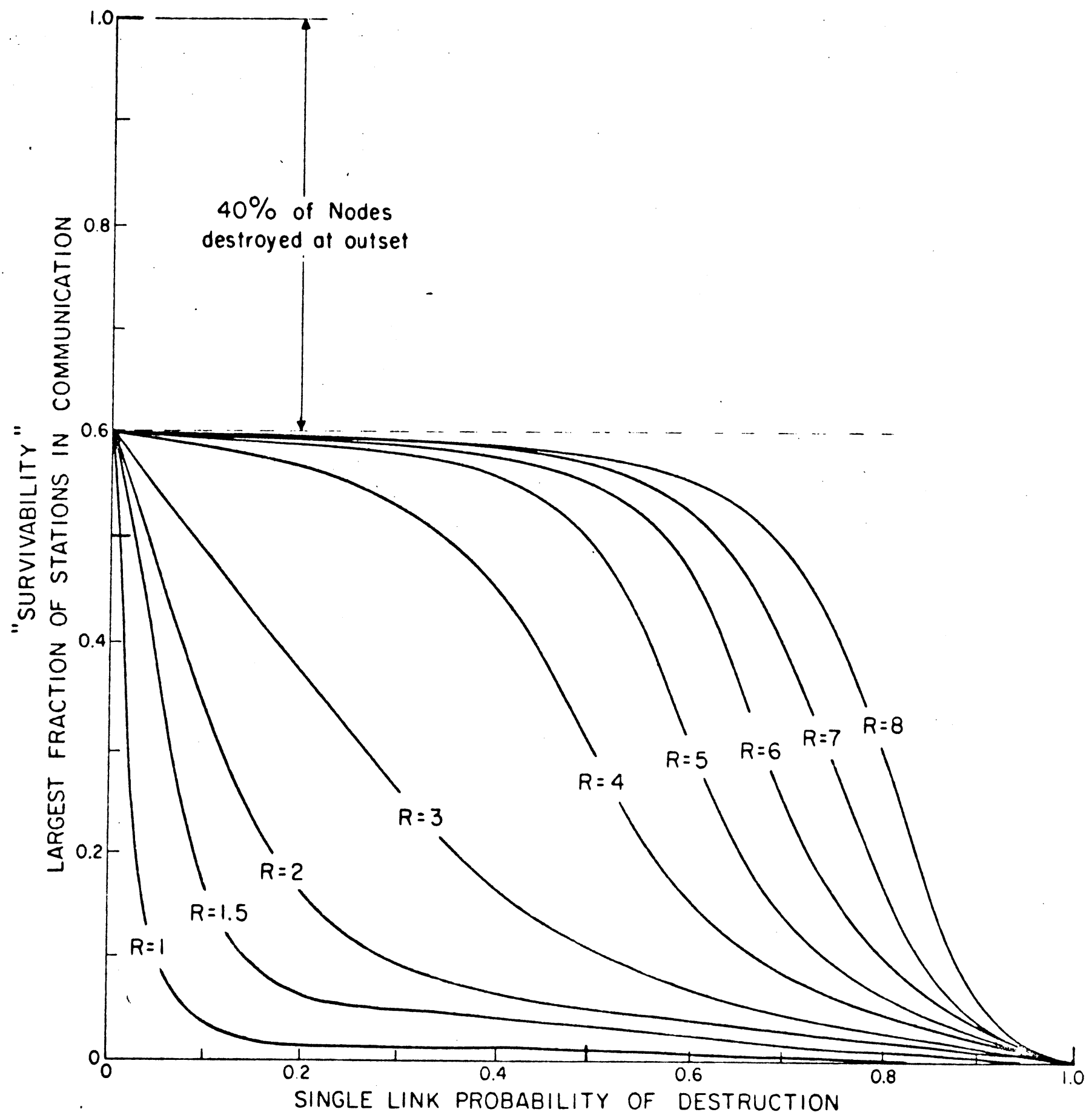

FIG. 6 - Perfect Switching in a Distributed Network - Sensitivity to Link Destruction After 40\% Nodes Are Destroyed. 
network almost as effectively as perfectly reliable links. Figure 7 examines the result of 100 trial cases in order to estimate the probability density distribution of system performance for a mixture of node and link failures. This is the distribution of cases for 20 percent nodal damage and 35 percent link damage.

\section{DIVERSITY OF ASSIGNMENT}

There is another and more common technique for using redundancy than in the method described above in which each station is assumed to have perfect switching ability. This alternative approach is called "diversity of assignment." In diversity of assignment, switching is not required. Instead, a number of independent paths are selected between each pair of stations in a network which requires reliable communications. But, there are marked differences in performance between distributed switching and redundancy of assignment as revealed by the following Monte Carlo simulation.

In the matrix of $\mathbf{N}$ separate stations, each ith station is connected to every $j$ th station by three shortest but totally separate independent paths $(i=1,2,3, \ldots, N$; $j=1,2,3, \ldots, N ; i \neq j)$. A raid is laid against the network. Each of the pre-assigned separate paths from the ith station to the jth station is examined. If one or more of the pre-assigned paths survive, communication is said to exist between the $i$ th and the $j$ th station. The criterion of 


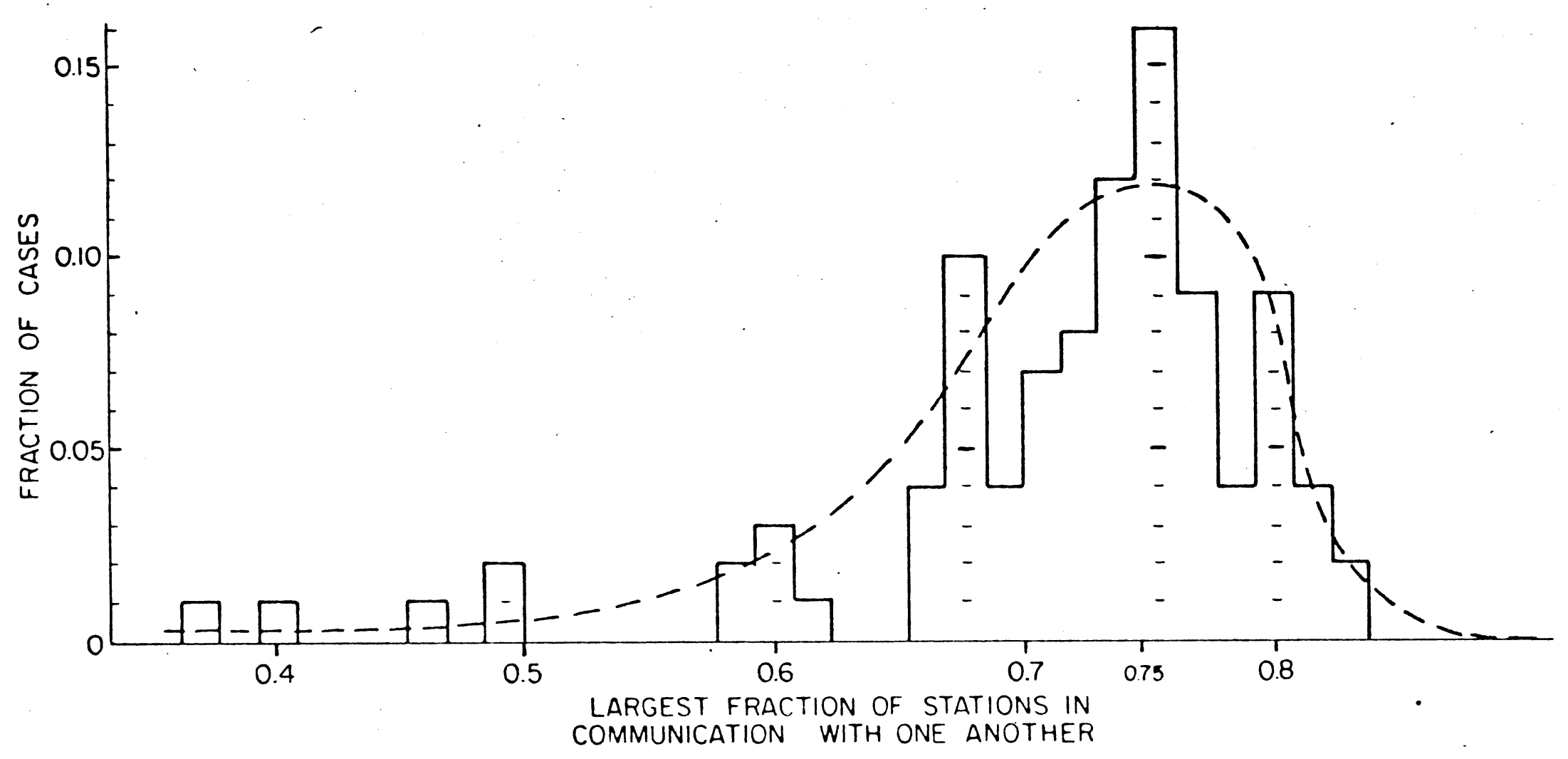

FIG. 7 - Probability Density Distribution of Largest Fraction of Stations in Communication Perfect Switching, $R=3,100$ Cases, $80 \%$ Node Survival, 65\% Link Survival. 
survivability used is the mean number of stations connected to each station, averaged over all stations.

Figure 8 shows, unlike the distributed perfect switching case, that there is a marked loss in communications capability with even slightly unreliable nodes or links. The difference can be visualized by remembering that fully flexible switching permits tho communicator the privilege of ex post facto decision of paths. Figure 8 emphasizes a key difference between some present day networks and the fully flexible distributed network we are discussing.

COMPARISON WITH PRESENT SYSTEMS

Present conventional switching systems try only a small subset of the potential paths that can be drawn on a gridded network. The greater the percentage of potential paths tested, the closer one approaches the performance of perfect switching. Thus, perfect switching provides an upper bound of expected system performance for a gridded network; the diversity of assignment case, a lower bound. Between these two limits lie systems composed of a mixture of switched routes and diversity of assignment.

Diversity of assignment is useful for short paths, eliminating the need for switching, but requires survivability and reliability for each tandem element in long haul circuits passing through many nodes. As every component in at least one out of a small number of possible paths 


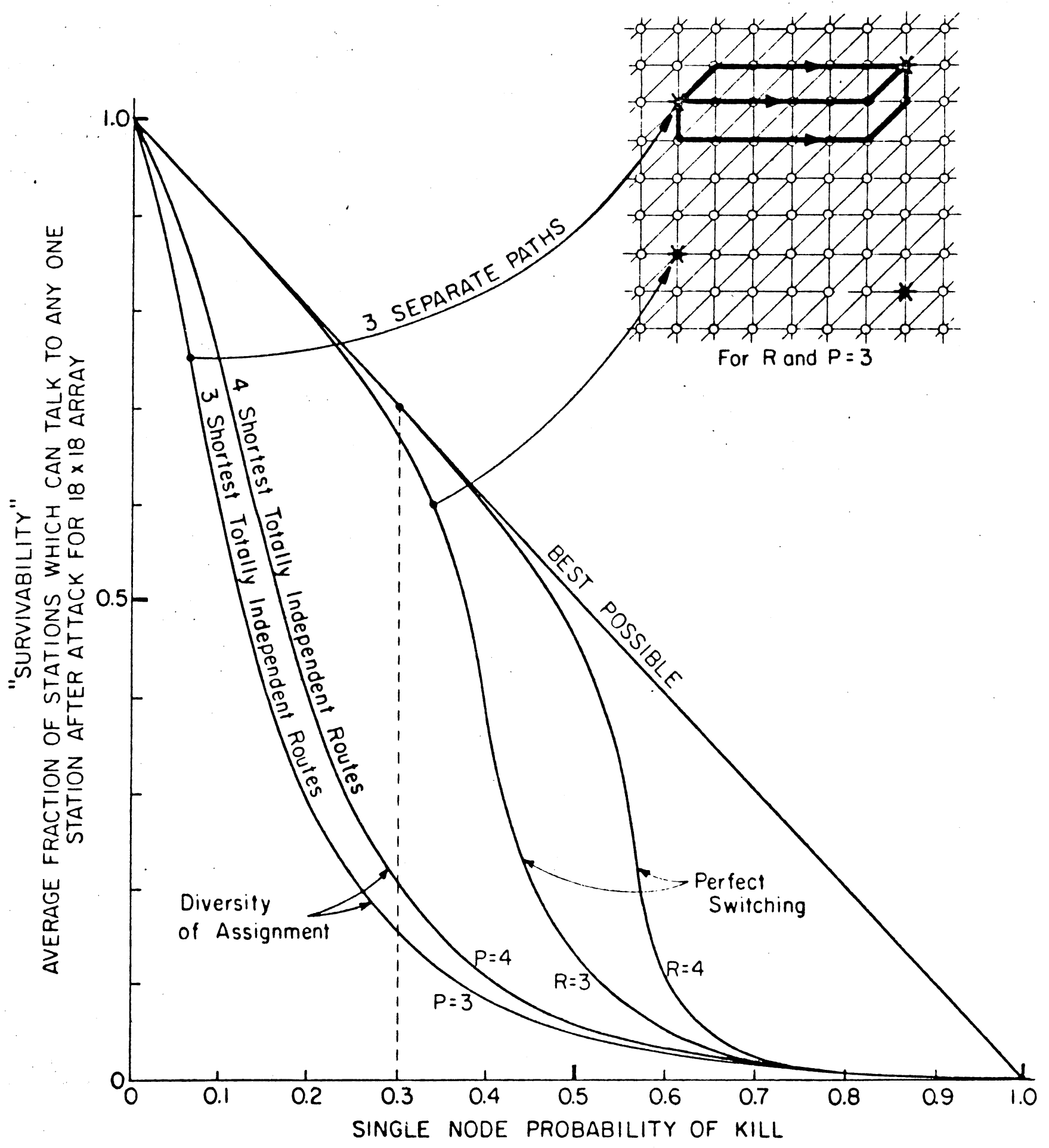

FIG. 8 - Diversity of Assignment vs. Perfect Switching in a Distributed Network. 
must be simultaneously operative, high reliability margins and full standby equipment are usual.

ON FUTURE SYSTEMS

We will soon be living in an era in which we cannot guarantee survivability of any single point. However, we can st1ll design systems in which system destruction requires the enemy to pay the price of destroying $n$ of $\mathrm{n}$ stations. If $\mathrm{n}$ is made sufficiently large, it can be shown that highly survivable system structures can be bullt--even in the thermonuclear era. In order to bullo such networks and systems we will have to use a large number of elements. We are interested in knowing how inexpensive these elements may be and still permit the system to operate reliably. There is a strone relationship between element cost and element reliability. To desien a system that must anticipate a worst-case destruction of both enemy attack, and normal system failures, one can combine the expected fallures expected by enemy attack together with the failures caused by normal reliability problems, provided the enemy does not know which elements are inoperative. Our future systems design problem is that of bulloing very reliable systems out of the described set of unreliable elements at lowest cost. In choosine the communications links of the future, digital links appear increasingly attractive by permitting low cost switching and low cost links. For example, if "perfect 
switching" is used, digital links are mandatory to permit tandem connection of many separately connected links without cumulative errors reachine an irreducible magnitude. Further, the signalling measures to implement highly flexible switching doctrines always require digits.

FUTURE LOW COST ALL-DIGITAL COMMUNICATIONS LINKS

When one designs an entire system optimized for dieits and high redundancy, certain new communications-link techniques appear more attractive than those common today.

A key attribute of the new media is that it permits formation of new routes cheaply, yet allows transmission on the order of a mililon or so bits per second, high enough to be economic, but yet low enough to be inexpensively processed with existing digital computer techniques at the relay station nodes. Reliability and raw error rates are secondary. The network must be built with the expectation of heavy damage, anyway. Powerful error removal methods exist.

Some of the communication construction methods that look attractive in the near future include pulse regenerative repeater line, "poor-boy" microwave, TV broadcast station digital transmission, and non-synchronous satellites.

\section{Prise Regenerative Repeater Iine}

Samuel B. Morse's regenerative repeater invention for amplifying weak telegraphic signals has recently been 
resurrected and transistorized. Morse's electrical relay permits amplification of weak binary telegraphic signals above a fixed threshold. Experiments by various organizations (primarily the Bell Telephone Laboratories) have shown that digital data rates on the order of 1.5 million bits per second can be transmitted over orainary telephone line at repeater spacings on the order of 6,000 feet for \#22 gage pulp paper insulated copper pairs. At present, up to 20 tandemly connected amplifiers have been used without retiming synchronization problems. There appears to be no fundamental reason why either lines of lower loss with corresponding further repeater spacing, or more powerful resynchronization methods cannot be used to extend link distances to in excess of 100 miles. Such distances would be desired for a possible national distributed network. Power to energize the minlature transistor amplifier is transmitted over the copper circuit itself.

"Poor-Boy" Microwave

While the price of microwave equipment has been declining, there are still untapped major savings. In an analog signal network we require a high degree of reliability and very low distortion for each tandem repeater. However, using digital modulation together with perfect switching we minimize these two expensive considerations from our planning. We would envision the use of almost mass-produced 
microwave crystal receiver/klystron oscillator units mounted on "telegraph poles" carrying commercial power. Relay station spacing would probably be on the order of 10+ miles. Further economies can be obtained by only a minimal use of standby equipment and reduction of fading margins. The ability to use alternate paths permits consideration of frequencies normally troubled by rain attenuation problems reducing the spectrum availability problem.

While this technique has not been fully examined, preliminary indications suggest that this may be the cheapest way of building large networks of the type to be described.

T. V. Stations

With proper siting of receiving antennas, broadcast television stations might be used to form additional high data rate links in emergencies.

Non-Synchronous Satelitites

The problem of building a reliable network using nonsynchronous satellites is somewhat similar to that of building a communications network with unreliable links. When a satellite is overhead, the link is operative. When a satellite is not overhead, the link is out of service. Thus, such links are highly compatible with the type of system to be described. 


\section{VARIABLE DATA RATE LINKS}

In a conventional circuit switched system each of the tandem links require matched transmission bandwidths. But, in the previous paper, * it was seen that in order to make fullest use of a digital link the post-error-removal data rate would have to vary as it is a function of noise level. The problem then is to build a communication network made up of links of variable data rate to use the communication resource most efficiently.

VARIABLE DATA RATE USERS

Not only will the links of a digital data transmission operate at a variable data rate, so will the users. Many digital transmission applications are highly intermittent in nature, with each potential network user varying his demand from instant to instant. For example, if one transmitted one line of a $60 \mathrm{w} . \mathrm{p} . \mathrm{m}$. teletype message over a high-data "express route" operating at 1,500,000 bits per second, a 1/3 millisecond burst would be sent every 12 seconds. Where high data rate transmission links serve many subscribers on a time division basis, both the user and the network links will appear to be operating at a highly variable data rate.

*See footnote, p. I. 
COMMON USER

In communications, as in transportation, it is most economic for many users to share a common resource rather than each to build his own system--particularly when supplying intermittent or occasional service. This intermittency of service is highly characteristic of digital communication requirements. Therefore, we would like to consider the interconnection, one day, of many all digital links to provide a resource optimized for the handling of data for many potential intermittent users--a new commonuser system.

Figure 9 demonstrates the basic notion. A wide mixture of different digital transmission links is combined to form a common resource divided among many potential users. But, each of these communications links could possibly have a different data rate. How can links of different data rates be interconnected?

\section{USE OF STAIDARD MESSAGE BLOCK}

Present common carrier communications networks, used for digital transmission, use links and concepts originally designed for another purpose--volce. These systems are built around a frequency division multiplexing linkto-link interface standard. The standard between links is that of data rate. Time division multiplexing appears so natural to data transmission that we might wish to 


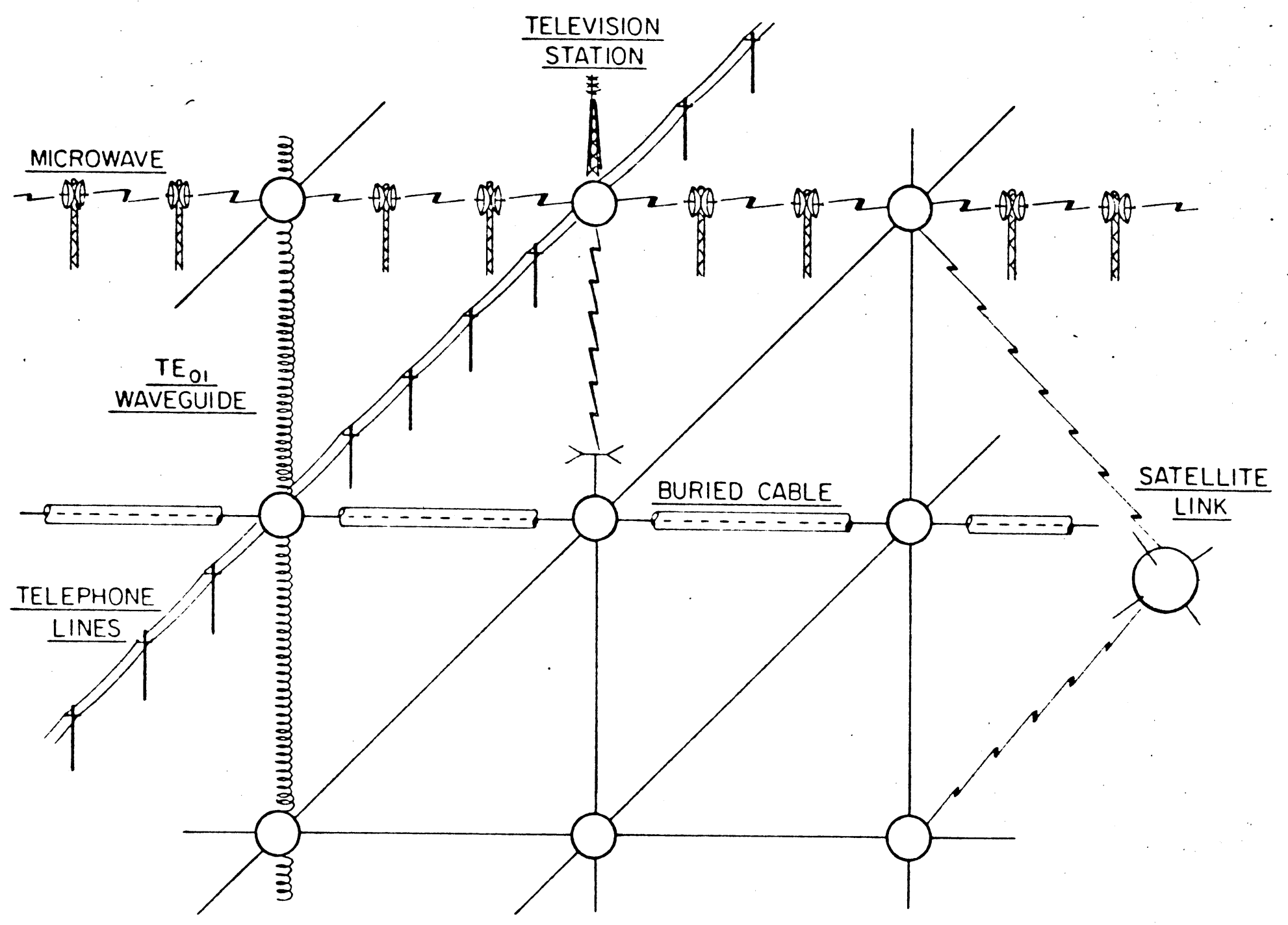

FIG. 9 - All Digital Network Composed of Mixture of Links 
consider an alternative approach--a standardized message block as a network interface standard. While a standardized message block is common in many computer-communications applications, no serious attempt has ever been made to use it as a universal standard. A universally standardized message block would be composed of perhaps 1024 bits. Most of the message block would be reserved for whatever type data is to be transmitted, while the remainder would contain housekeeping information such as error detection and routing data, as in Fig. 10 .

As we move to the future, there appears to be an increasing need for a standardized message block for our all-digital communications networks. As data rates increase, the velocity of propagation over long links becomes an increasingly important consideration. * We soon reach a point where more time is spent setting the switches in a conventional circuit switched system for short holding-time messages than is required for actual transmission of the data.

Most importantly, standardized data blocks permit many simultaneous users each with widely different bandwidth requirements to economically share a broadband network made up of varied data rate links.

*3000 miles at $\simeq 150,000 \mathrm{miles} / \mathrm{sec} . \simeq 50 \mathrm{milliseconds}$ transmission time, T. 1024 -bit message at $1,500,000 \mathrm{bits} / \mathrm{sec} . \simeq 2 / 3 \mathrm{milli}-$ second message time, $M$. 

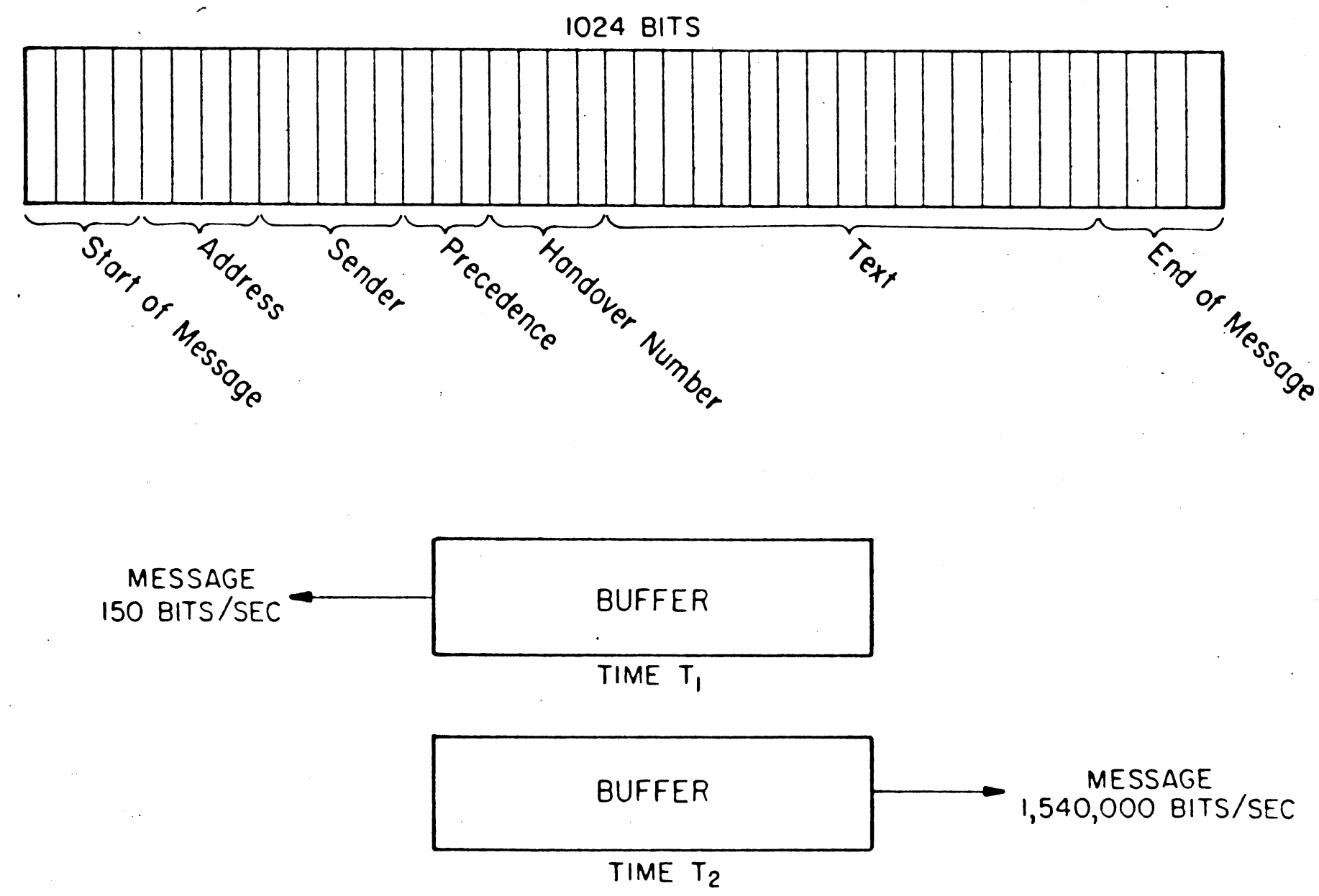

FIG. 10 - Message Block 
The standardized message block simplifies construction of very high speed switches. Every user connected to the network can feed data at any rate up to a maximum value. The user's traffic is stored until a full data block is received by the first station. This block is rubber stamped with a heading and return address, plus additional housekeeping information. Then, it is transmitted into the network.

\section{SWITCHING}

In order to build a network with the survivability properties shown in Fig. 4, we must use a switching scheme able to find any possible path that might exist after heavy damage. The routing doctrine should find the shortest possible path and avoid self-oscillatory or "ringaround-the-rosey" switching.

We shall explore the possibilities of building a "real-time" data transmission system using store and forward techniques. The high data rates of the future carry us into a hybrid zone between store-and-forward and circuit switching. The system to be described is clearly store-and-forward if one examines the operations at each node singularly. But, the network user who has called up a "virtual connection" to an end station and has transmitted messages across the United States in a fraction of a second might also view the system as a black box providing 
an apparent circuit connection across the U.S. There are two requirements that must be met to build such a quasireal time system. First, the in-transit storage at each node should be minimized to prevent undesirable time delays. Secondly, the shortest instantaneously available path through the network should be found with the expectation that the status of the network will be rapidly changing. Microwave would be subject to fading interruptions and there would be rapid moment-to-moment variations in input loading. These problems place difficult requirements upon the switching. However, the development of digital computer technology has advanced so rapidly that it now appears possible to satisfy these requirements by a moderate amount of digital equipment. What is envisioned is a network of unmanned digital switches implementing a self-learning policy at each node so that overall traffic is effectively routed in a changing environment--without need for a central and possibly vulnerable control point. One particularly simple routing scheme examined is called the "hot-potato" heuristic routing doctrine and will be described in detail.

Torn-tape telegraph repeater stations and our mail system provide examples of conventional store-and-forward switching systems. In these systems, messages are relayed from station-to-station and stacked until the "best" outgoing link is free. The key feature of store-and- 
forward transmission is that it allows a high line occupancy factor by storing so many messages at each node that there is a backlog of traffic awaiting transmission. But, the price for link efficiency is the price paid in storage capacity and time delay. However, it was found that most of the advantages of store-and-forward switching could be obtained with extremely little storage at the nodes.

Thus, in the system to be described, each node will attempt to get rid of its messages by choosing alternate routes if its preferred route is busy or destroyed. Each message is regarded as a "hot potato," and the nodes are not wearing gloves. Rather than hold the "hot potato," the node tosses the message to its neighbor, who will now try to get rid of the message.

\section{THE POSTMAN}

The switching process in any store-and-forward system is analogous to a postman sorting mail. A postman sits at each switching node. Messages arrive simultaneously from all links. The postman records bulletins describing the traffic loading status for each of the outgoing links. With proper status information, the postman is able to determine the best direction to send out any letters. So far, this mechanism is general and applicable to all storeand-forward communication systems. 
HOT-POTATO HEURISTIC ROUTING DOCTRINE

To achieve real-time operation it is desirable to respond to change in network status as quickly as possible so we shall seek to derive the network status information directly into each message block.

Each standardized message block contains a "to" address, a "from" address, a handover number tag, and error detecting bits together with other housekeeping data. The message block is analogous to a letter. The "from" address is equivalent to the return address of the letter.

The handover number is a tag in each message block set to zero upon initial transmission of the message block into the network. Every time the message block is passed on, the handover number is incremented. The handover number tag on each message block indicates the length of time in the network or path length. This tag is somewhat analogous to the cancellation date of a conventional letter.

\section{INDUCTIVE DETERMINATION OF BEST PATH}

Assuming symmetrical bi-directional links, the postman can infer the "best" paths to transmit mail to any station merely by looking at the cancellation time or the equivalent handover number tag. If the postman sitting in the center of the United States received letters from San Francisco, he would find that letters from San Francisco arriving from channels to the west would come in with later cancellation dates than if such letters had 
arrived in a roundabout manner from the east. Each letter carries an implicit indication of its length of transmission path. The astute postman can then deduce that the best channel to send a message to San Francisco is probably the link associated with the latest cancellation dates of messages from San Francisco. By observing the cancellation dates for all letters in transit, information is derived to route future traffic. The return address and cancellation date of recent letters is sufficient to determine the best direction to which to send subsequent letters.

THE HANDOVER NUNBER TABLE

While cancellation dates could conceivably be used on digital messages, it is more convenient to think in terms of a simpler digital analogy--a tag affixed to each message and incremented every time the message is relayed. Figure 11 shows the handover table located in the memory of a single node. A row is reserved for each major station of the network allowed to generate traffic. A column is assigned to each separate link connected to a node. As it was shown that redundancy levels on the order of four can create extremely "tough" networks and additional redundancy brought little, only about eight columns are really needed. 


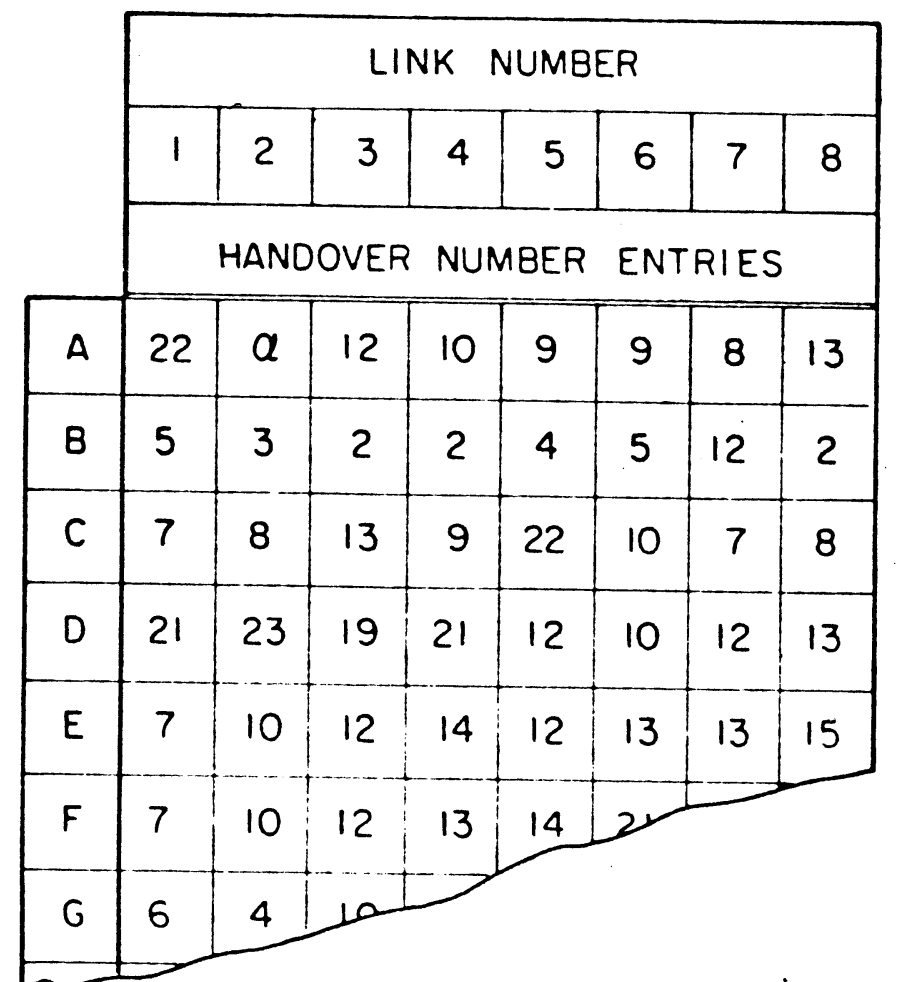

\begin{tabular}{|c|c|c|c|c|}
\hline \multicolumn{5}{|c}{ BEST CHOICE } \\
\hline Ist & 2nd & 3rd & 4 Ih & $\vdots \cdots$ \\
\hline \multicolumn{4}{|c|}{ LINK } & \multicolumn{3}{|c}{ NUMBER } & for \\
\hline DECISION & CHOICE \\
\hline 7 & 5 & 6 & 4 & $\vdots$ \\
\hline 3 & 4 & 8 & 2 & $\vdots$ \\
\hline 1 & 7 & 2 & 8 & $\vdots$ \\
\hline 6 & 5 & 7 & 8 & $\vdots$ \\
\hline 1 & 2 & 3 & 5 & $\vdots$ \\
\hline 1 & 2 & 3 & 4 & $\vdots$ \\
\hline 5 & 2 & 1 & 6 & \\
\hline
\end{tabular}
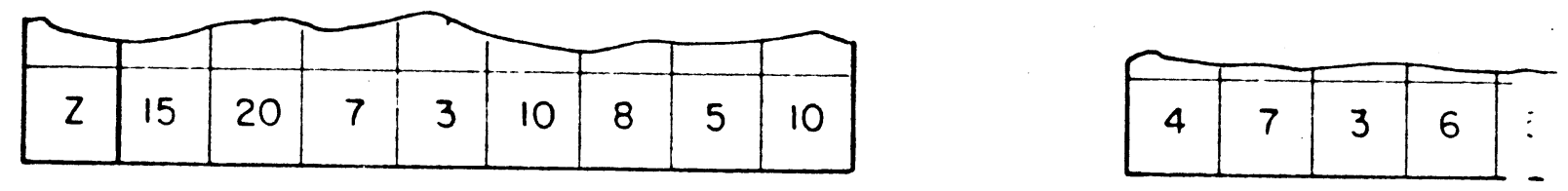

FIG. II - The Handover Number Table 


\section{PERFECT IEARNING}

If the network used perfectly reliable, error free links, we might fill out our table in the following manner. Initially, set entries on the table to high values. Examine the handover number of each message arriving on each line for each station. If the observed handover number is less than the value already entered on the handover number table, change the value to that of the observed handover number. If the handover number of the message is greater than the value on the table, do nothing. After a short time this procedure will shake down the table to indicate the path length to each of the stations over each of the links connected to neighboring stations. This table can now be used to route new traffic. For example, if one wished to send traffic to station $C$, he would examine the entries for the row listed for station $\mathrm{C}$ based on traffic from $C$. Select the link corresponding to the column with the lowest handover number. This is the shortest path to $C$. If this preferred link is busy, do not wait, choose the next best link that is free. 
DIGITAL SIMULATION OF PERFECT LEARNING

1 This basic routing procedure was tested by a Monte Carlo simulation of a $7 \times 7$ array of stations.* All tables were started completely blank to simulate a worst-case starting condition where no station knew the location of any other station. Within $\frac{1}{2}$ second of simulated real world time, the network had learned the locations of all connected stations and was routing traffic in an efficient manner. The mean measured path length compared very favorably to the absolute shortest possible path length under various traffic loading conditions. Preliminary results indicate that network loadings on the order of 50 per cent of link capacity could be inserted without undue increase of path length. When local busy spots occur in the network, locally generated traffic is intermittently restrained from entering the busy points while the potential traffic fams clear. Thus, to the user, the network appears to be a variable data rate system. If the network is carrying light traffic, any new input line into the network would accept full traffic up to 1.5 million bits per second. But, if every station had heavy traffic and the network became heavily loaded, the total allowable input data rate from any single station in the network might

* Paul Baran and Sharla Boehm, Simulation of a Hot Potato Routing Doctrine (U), The RAND Corporation, RM-3103, (In preparation). 
drop to perhaps 0.5 million bits per second. The absolute minimum guaranteed data capacity into the network from any station is a function of the location of the station in the network, redundancy level, and the mean path length of transmitted traffic in the network. The "choking" of input procedure has been simulated in the network and no signs of instability under overload noted. It was found that most of the advantage of store-and-forward transmission can be provided in a system having relatively little memory capacity. The network "guarantees" delivery of all traffic that it has accepted from a user.

FORGETTING AND IMPERFECT LEARNING

We have briefly considered network behavior when all links are working. But, we are also interested in determining network behavior with real world links--some destroyed, while others are being repaired. The network can be made rapidly responsive to the effects of destruction, repair, and transmission fades by a slight modification of the rules for computing the values on the handover number table. In the previous example, the lowest handover number ever encountered for a given origination, or "from" station, and over each link, was the value recorded in the handover number table. But, if some links had failed, our table would not have responded to the change. Thus, we must be more responsive to recent measurements 
than old ones. This effect can be included in our calculation by the following policy. Take the most recently measured value of handover number; subtract the previous value found in the handover table; if the difference is positive, add a fractional part of this difference to the table value to form the updated table value. This procedure merely implements a "forgetting" procedure--placing more belief upon more recent measurements and less on old measurements. This device would, in the case of network damage, automatically modify the handover number table entry so as to exponentially and asymptotically approach the true shortest path value. If the difference between measured value minus the table value is negative, the new table value would change by only a fractional portion of the recently measured difference.

This implements a form of sceptical learning. Learning will take place even with occasional errors. Thus, by the simple device of using only two separate "learning constants," depending whether the measured value is greater or less than the table value, we can provide a mechanism that permits the network routing to be responsive to varying loads, breaks, and repairs. This learning and forgetting technique has been simulated for a few limited cases and was found to work well. 
ADAPTATION TO ENVIRONMENT

This simple simultaneous learning and forgetting mechanism implemented independently at each node causes the entire network to suggest the appearance of an adaptative system responding to gross changes of environment in several respects, without human intervention. For example, consider self-adaptation to station location. A station, Able, normally transmitted from one location in the network, as shown in Fig. 12 (a). If Able moved to the location shown in Fig. 12 (b), all he need do to announce his new location is to transmit a few seconds of dummy traffic. The network will quickly relearn the new location and direct traffic toward $\mathrm{Able}$ at his new location. The links could also be cut and altered, yet the network would relearn. Each node sees its environment through myopic eyes by only having links and link status information to a few neighbors. There is no central control; only a simple local routing policy is performed at each node, yet the overall system adapts.

\section{LOWEST COST PATH}

We seek to provide the lowest cost path for data to be transmitted between users. When we consider complex networks, perhaps spanning continents, we encounter the problem of building networks with links of widely different data rates. How can paths be taken to encourage 

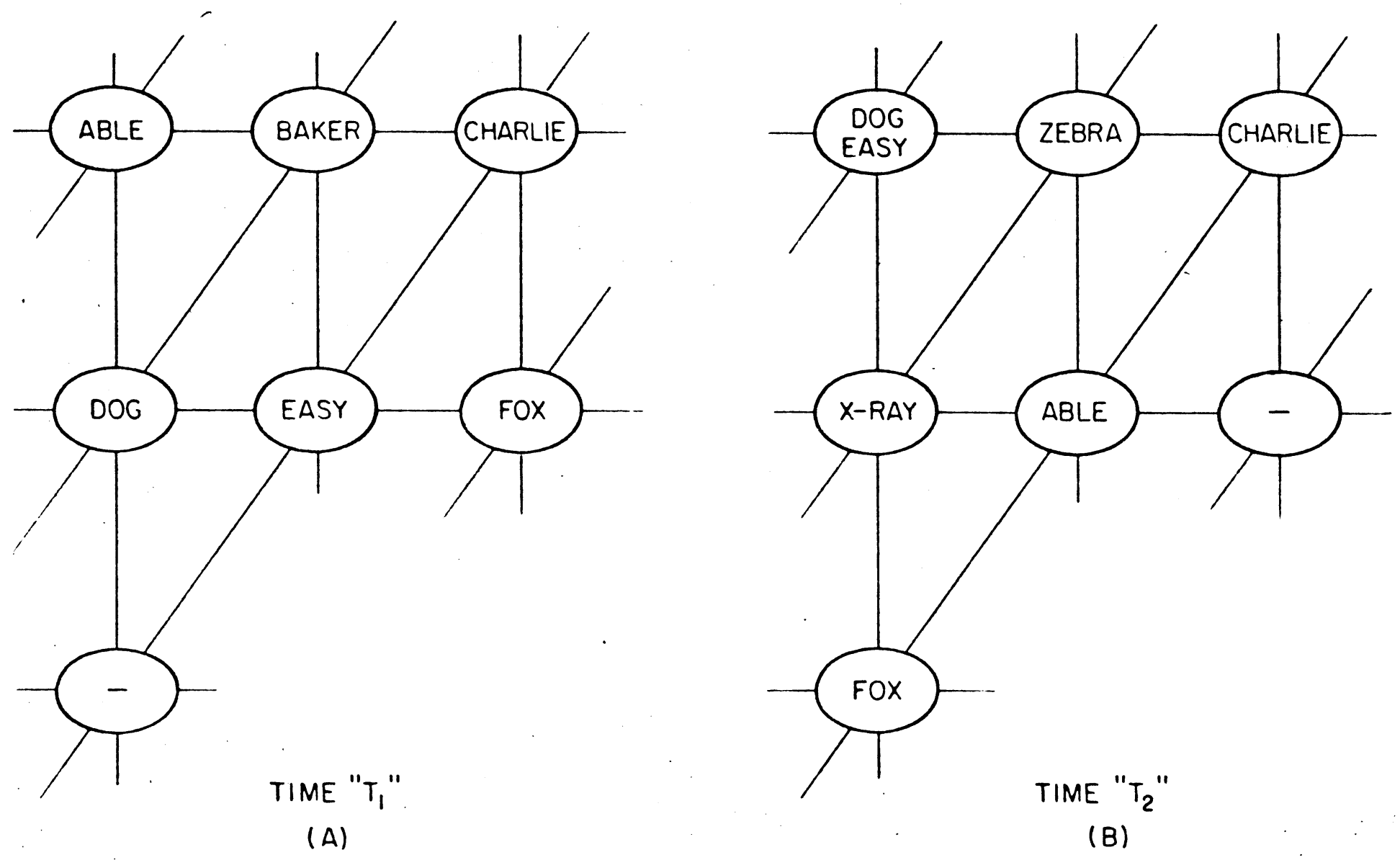

FIG. 12-Adaptability to Change of User Location 
most use of the least expensive links? The fundamentally simple adaptation technique can again be used. Instead of incrementing the handover by a fixed amount, each time a message is relayed, set the increment to correspond to link cost/bit of the transmission link. Thus, instead of the "instantaneously shortest non-busy path" criterion, the path taken will be that offering the cheapest transportation cost from user to user that is available. The technique can be further extended by placing priority and cost bounds in the message block itself, permitting certain users more of the communication resource during periods of heavy network use.

\section{WHERE WE STAND TODAY}

Although it is premature at this time to know all the problems involved in such a network and understand all costs, there are reasons to suspect that we may not wish to build future digital communication networks exactly the same way the nation has built its analog telephone plant.

There is an increasingly repeated statement made that one day we will require more capacity for data transmission than needed for voice. If this statement is correct, then it would appear prudent to broaden our planning consideration to include new concepts for future data network directions. Otherwise, we may stumble into being boxed in with the 
uncomfortable restraints of communications links and switches originally designed for high quality analog transmission. New digital computer techniques using redundancy make cheap unreliable links potentially usable. Some sort of a switched network compatible with these links appears appropriate to meet this new upcoming demand for digital service.

of course, we could use our existing circuit switching techniques. But, a system with greater capacity than the long lines of telephone plants might best be designed for such data transmission and survivability at the outset. Such a system should economically permit switching of very short blocks of data from a large number of users simultaneously with intermittent large volumes among a smaller set of points. Considering the size of the market there appears to be an incommensurately small amount of thinking about a national data plant designed primarily for data.

Is it time now to start thinking about a new and possibly non-existant public utility, a common user digital data communication plant designed specifically for the transmission of digital data among a large set of subscribers?

Is it time to consider the detailed format of a standard message block as a possible new data standard of the future? 